\title{
Comparison between Effect of Sildenafil Citrate and Tramadol on Testis and Sperm Quality of Male Rats
}

\author{
Reham E. Masoud \\ Department of Clinical Pharmacology, Faculty of Medicine, Port Said University, Egypt
}

\begin{abstract}
Background: Nowadays tramadol is becoming abused more popular among teens in most countries worldwide; especially between males. Sildenafil citrate is an oral medication widely used to treat erectile dysfunction. Aim: to evaluate and compare effects of these two drugs on count and morphology of sperms in male rats. Materials and Methods: The study groups included normal control, sildenafil citrate treated and tramadol treated group, each group included 10 rats. Serum testosterone level and testicular histopathology were evaluated. Serum testosterone was measured by ELISA, sperm count and morphology were assessed and testicular histopathology was assessed after sacrifice. Results: The present work revealed that treatment with sildenafil citrate and tramadol separately caused a significant decreased count of sperms and increased abnormal forms as compared to normal ( $p \leq 0.05)$. There was also significant decrease in testosterone level in tramadol group as compared to both normal and sildenafil treated group ( $p$ value $\leq 0.05$ ). Histopathology of testes showed reduction of spermatogenic cells number and height and increase interstitial tissue between tubules and in tramadol treated group, loss of most spermatogenic cells, degenerative changes, disturbed architecture of seminiferous tubules and edema of interstitial tissue. Conclusions: The study concluded that these two drugs impaired testicular function in male rats to varying degrees, tramadol caused more significant impairment in all parameters measured than sildenafil citrate and recommended limitation the use of these two drugs if possible and necessity of designing a national awareness campaign to the public to spotlights on the hazards of tramadol abuse on sexual function.
\end{abstract}

Key words: sildenafil, tramadol, nitric oxide, testosterone.

\section{Introduction}

Sildenafil citrate is the one of the drugs which were approved for the treatment of erectile dysfunction, its mechanism of action is through inhibition of phosphodiesterase 5 (PDE5), the enzyme that metabolizes cyclic guanosine monophosphate (c-GMP) in the corpus cavernosum ${ }^{(1)}$. The penile erection is dependent on the release of nitric oxide (NO) in the corpus cavernosum. NO increase levels of (cGMP) which results during the smooth muscle relaxation and inflow of blood, and sildenafil enhanced the effect of NO by inhibiting PDE5 which is responsible for degradation of CGMP corpus cavernosum(2). It was proved that human sperm cells have PDE isoforms which differ from PDE1 and PDE4, and that the in-vitro inhibition of sperm PDE1 and PDE4 isoenzymes by specific inhibitors stimulates acrosome reaction and sperm motility(3). Sildenafil citrate is an effective drug for erectile dysfunction due to 
organic causes(4), however side effects have been reported such as flushing, headache, congestion and dyspepsia, occur due to the systematic vasodilator effect of the drug although no clinically adverse homodynamic effects were observed when sildenafil was used by healthy men ${ }^{(5)}$. Systolic and diastolic systemic arterial pressure were shown to be decreased $6 \%$ and cardiac output was also shown to be decreased $11 \%$ from the baseline during the post sildenafil exercise test ${ }^{(6)}$. Tramadol is a centrally active synthetic opioid analgesic that is used extensively. Its mode of action is not completely understood, but two acceptable complementary mechanisms are binding to mu opioid receptors (MOR) and inhibition of reuptake of noradrenaline and serotonin $^{(7)}$. From literature, multiple cases of toxicity and abuse of tramadol have been reported. The main symptoms of toxicity include central nervous system depression, nausea and vomiting, tachycardia, and seizures ${ }^{(8)}$. Fatal cases have been reported because of tramadol overdose. In those instances, death has been attributed to cardiopulmonary arrest and hepatic failure as well as hypoglycemia(9). The tramadol absorption is more than morphine reached to about $95-100 \%$ and absorbed rapidly in the small intestine. and reached highest peak after $5 \mathrm{hrs}^{(10)}$. It is widely distributed throughout the body, especially liver and kidneys ${ }^{(11)}$. There is no work concerning the reproduction function. These two drugs are widely used by adults and youth so the present work was conducted to assess and compare the reproductive effect of these two drugs on some of sperm characteristics (sperm count, sperm malformation) and testis architecture of male rats.

\section{Material and Methods}

This experimental study was conducted in the Suez Canal University, Faculty of Medicine. Male albino rats weighing 200-250 g with 8 weeks of old were used. The animals were divided into i) control group $(n=10)$ treated with $0.9 \% \mathrm{NaCl}$ for 4 weeks oral. ii) The first treated group $(\mathrm{n}=10)$ treated with (50 mg/kg body weight) sildenafil citrate for 4 weeks orally ${ }^{(12)}$. iii) The second treated group $(\mathrm{n}=10)$ treated with (40mg/kg) tramadol for 4 weeks orally ${ }^{(13)}$. Animals were purchased from the Egyptian Organization for Biological Products and Vaccines (Egypt), and allowed free access to food and water ad libitum. They were kept under constant conditions with $12 / 12 \mathrm{~h}$ light/dark cycles and left for acclimatization for one week before the start of the study. The care and handling of the animals were in accordance with the Animal Care and Use Committee at the Suez Canal University and the National Institutes of Health guide for the care and use of laboratory animals (Maryland, USA). All efforts were made to minimize animal suffering and to reduce the number of animals used. Drugs and Chemicals: All drugs and chemicals were purchased from Sigma Aldrich, USA. The doses of drugs were based on previous studies and were nearly the same as the dose calculated according to Paget \& Barnes (1964) $)^{(14)}$, where the human daily dose was multiplied by the rat conversion factor (0.018). At the end of treatment, the animals of study groups were anaesthetized by giving injection Nembutol $30 \mathrm{mg}$ per kg, intraperitoneally. The heart was exposed by thoracotomy. The needle of the blood transfusion set was introduced into the left ventricle (apex) and a nick was made in the right atrium and their testis were incised. they were fixed in $10 \%$ phosphate buffered formalin ( $\mathrm{pH} \mathrm{7.4)}$ for routine histological investigation. The study tested the effect of the used drugs on the following: i) Serum testosterone: blood samples were collected after sacrifice directly from the heart and blood serum was separated. By using ELISA method, testosterone concentration was measured in 
study groups. ii) Sperm count and morphology: The caudal epididymis was cut and weighed. The epididymis was removed, cleaned off from the epidydimal fat pad, minced in $1 \mathrm{ml}$ of pre-warmed $\left(35^{\circ} \mathrm{C}\right)$ phosphate-buffered saline (PBS, $5 \mathrm{ml} \mathrm{g-1}$ testis $\mathrm{pH} 7.35)$ and filtered through $80 \mu \mathrm{m}$ poresize mesh. The filtrate was used for the evaluation of epidydimal sperm parameters. A cell suspension was prepared by macerating the cauda in $1.0 \mathrm{ml}$ of $0.85 \% \mathrm{sa}$ line. The cell suspension was kept for 24 hrs. at $40^{\circ} \mathrm{C}$. The suspension was then filtered through a double gauze layer and an aliquot of the sample was used for sperm count in a Neubauer hemocytometer. An aliquot of the epidydimal sperm suspension was smeared and stained with hematoxylin and eosin and then examined under a light microscope (Olympus, Japan) at magnification of $40 X$. The head and tail abnormalities (200 sperms per smear) were recorded(15). iii) Testicular histopathology: The testis was dissected out, cut into small slices, and fixed in 10\% formaldehyde buffer for $24 \mathrm{hrs}$. The tissues were washed free of $10 \%$ formaldehyde and stored in $70 \%$ alcohol until being embedded. The tissues were dehydrated in alcohol series and embedded in paraffin. Tissue sections of $5 \mathrm{~mm}$ thickness were prepared and placed on glass slides. Sections were stained with hematoxylin \& eosin, mounted in mounting medium and examined under the light microscope. For each testis several cross sections composing of 20-50 tubule sections were examined for signs of interstitial edema, somniferous tubule degeneration, and congestion ${ }^{(15)}$.

\section{Statistical analysis}

Data were checked, coded, entered and analyzed using SPSS (version 17.0 software). The results were expressed as means \pm SD on Microsoft Excel 8.o. Statistical analysis was performed using one way analysis of variance (ANOVA) followed by Tukey's test post hoc analysis. Values were considered statistically significant at $\mathrm{P} \leq 0.05$.

\section{Results}

1-Effect of drugs on sperm count: Long term administration of sildenafil citrate in male rats caused a significant reduced number of sperms compared with normal control group. Treatment with tramadol caused significant decrease in number of sperms compared to normal and sildenafil treated group (figure 1). 2-Effect of drugs on sperm morphology: The treatment of the male rats with sildenafil citrate revealed a significant increase in abnormal sperms compared with control group. Treatment with tramadol caused significant increase number of abnormal forms of sperms compared to normal control and sildenafil treated group (figure 1). 3-Effect of drugs on serum testosterone: There was significant decrease in serum testosterone level in tramadol group as compared to normal and sildenafil group as shown in Figure.2.

\section{Discussion}

In the present study, we investigated the effect of administration of sildenafil citrate and tramadol on sperm count, sperm malformations and testicular histopathological changes of male rats. Sildenafil citrate caused sperm abnormality (decrease number of sperms and increase of sperm's malformations) which supported by the histopathological changes in testis (hypertrophy cells, necrosis of seminiferous tubules, destruction of testis and presence of inflammatory cells). The very limited data available on the effects of sildenafil citrate on seminal parameters reveals that the oral administration of sildenafil citrate has some role on seminal parameters in the patients of erectile dysfunction. 


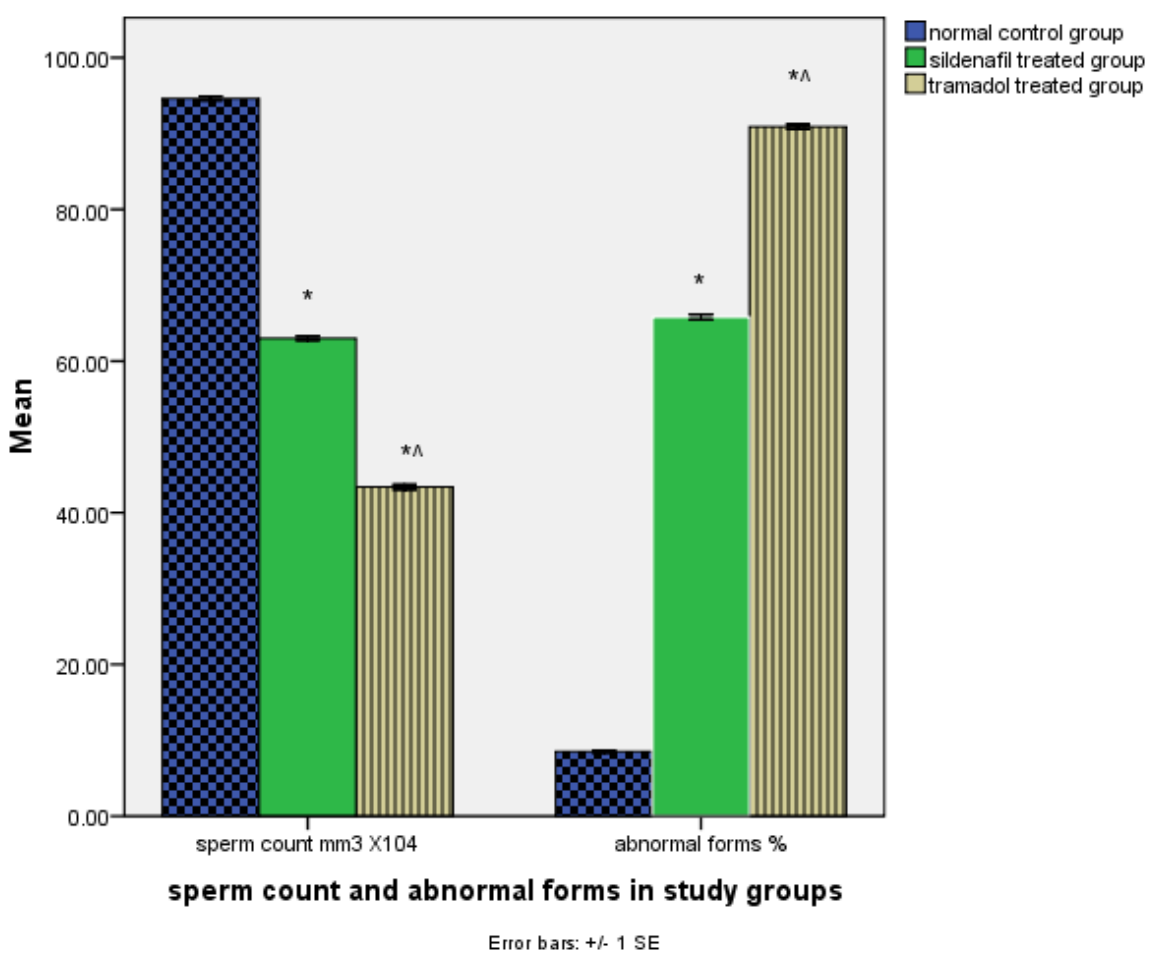

Figure 1: Effects of study drugs on sperm count and abnormal forms in study groups *=A significant difference compare with control group at $\mathrm{P} \leq 0.05 ;^{\wedge}=\mathrm{A}$ significant difference compare with group treated with sildenafil citrate at $P \leq 0.05$.

At least two distinct PDE isoforms (PDE1 and PDE4) have been demonstrated to be present in human sperm cells. Specific inhibition of PDE1 and PDE4 by 8-methoxy-isobutyl-methylxanthine and rolipram selectively stimulates the acrosome reaction and sperm motility respectively (3). Another study showed that sildenafil had no statistically significant effect on sperm motility, count or density, the percentage of abnormal sperms, or the percentage of living sperm when 17 healthy male volunteers aged 19-34 years were randomized to receive a single dose of $100 \mathrm{mg}$ dose of sildenafil for two periods and a single dose of placebo for two periods, with each period separated by a minimum of 5-7 days ${ }^{(16)}$. Other studies found the minimal effect of sildenafil on sperm motility and count in men of reproductive age. In the study of 20 healthy male volunteers, found no difference in sperm number, progressive motility or morphologic abnormalities between semen samples obtained 1 hour after taking a $100 \mathrm{mg}$ dose of sildenafil or double blind placebo ${ }^{(17-18)}$. Sildenafil had provoked tubular and interstitial histological alterations of the seminiferous tubules, increased Leydig cells cellularity, tubular degeneration, which finally might lead to complete arrest of spermatogenesis, but SC has no effect on epididymis histology ${ }^{(19)}$. This study detected significant association between tramadol administration and impaired quality sperm parameters as compared to normal control and more significant impaired quality as compared to sildenafil group. The results of sperm quality analysis showed a significant decrease in sperm concentration, motility and vitality that indicate the possibility of adverse effects of long term administration of tramadol on epidydimal spermatozoa of rats. in tramadol treated group, there was loss of most spermatogenic cells, degenerative changes, disturbed architecture of seminiferous tubules and edema of interstitial tissue. 


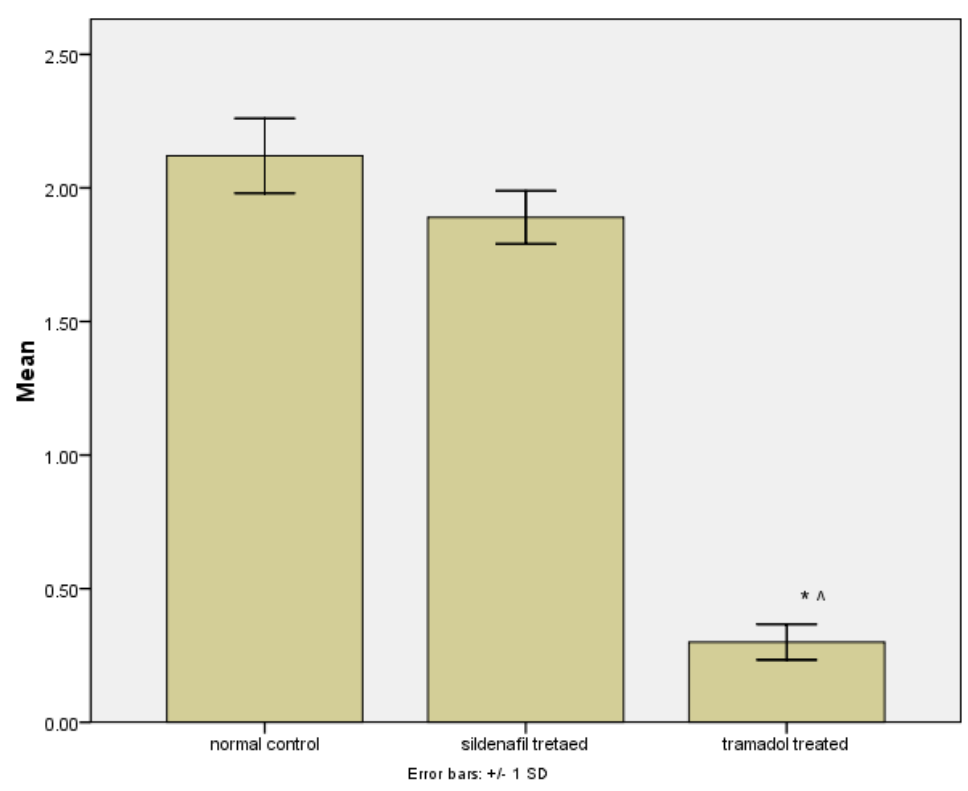

Figure 2: The mean plasma concentrations of testosterone in IU/I in different experiment groups compared with the control group $(*=$ A significant difference compare with control group at $\mathrm{P} \leq 0.05 ;^{\wedge}=\mathrm{A}$ significant difference compare with group treated with sildenafil citrate at $\mathrm{P} \leq 0.05$ ).

In previous studies, it has been showed that narcotics markedly reduce the structural and functional integrity of the secondary sex organs by causing pronounced reduction in serum testosterone levels. These drugs inhibit the secretion of luteinizing hormone ( $\mathrm{LH}$ ) by acting either in the hypothalamus or directly in the pituitary gland, which leads to reduced serum testosterone levels ${ }^{(20)}$. Reuhl et al., showed thickening of the basement membrane of seminiferous tubules, reduction of tubular diameter and height of the germinal epithelium, cell maturation arrest, and highest proportion of interstitial tissue in drug abusers $^{(21)}$. It is known that changes in the thickness of the basement membrane can impair testicular metabolism, and thus promotes enhanced germinal cell hypoplasia and tubular atrophy. The extent of testicular damage is closely related to the duration of drug consumption ${ }^{(22)}$. Administration of amphetamine compounds induces apoptosis in the seminiferous tubules in testes of mice, and changed testes histopathology in experimental animals(23). In this study, there was significant decrease in serum testosterone in tramadol treated group as compared to normal and sildenafil treated group, this may be due to degeneration of testicular tissue observed in histopathology. Recently chronic administration of tramadol on testicular function was investigated in the study of Ahmed and $\operatorname{Kurkar}^{(24)}$. In their study, the rat received $40 \mathrm{mg} / \mathrm{kg}$ of tramadol as subcutaneous for 8 weeks. Their result revealed that tramadol increased the expression of endothelial nitric oxide synthase in testicular tissues. Also, tramadol caused decrease in sperm count, motility, and numbers of primary spermatocytes, rounded spermatid and leydig cells. They concluded that long term administration of tramadol affects the testicular function of adult male rats and these effects may be through overproduction of $\mathrm{NO}$ and oxidative stress induced by this drug. 


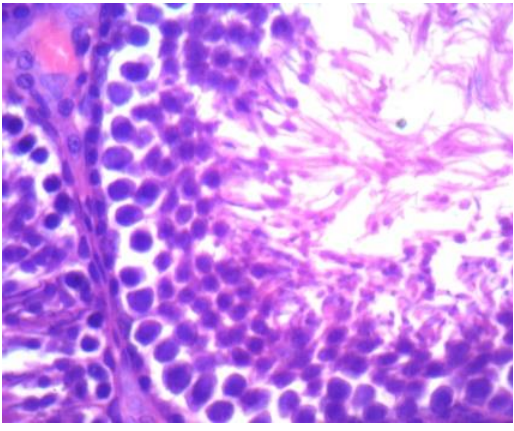

A normal control

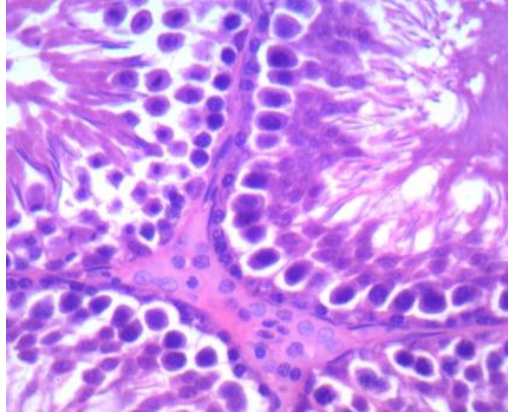

B sildenafil treated

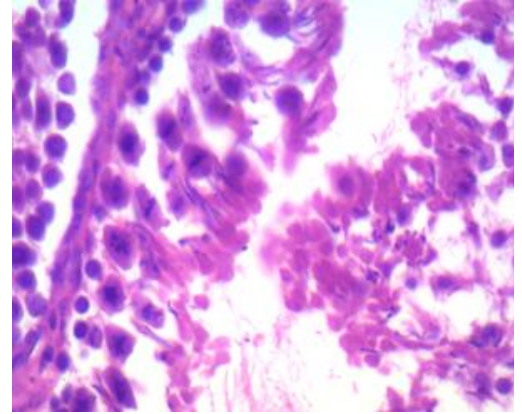

C tramadol treated

Figure 2. Histopathology of testis of study groups

Normal group testis showing normal seminiferous tubules with normal arrangement pattern of spermatogenic cells and interstital cells, sildenafil treated group showed reduction of spermatogenic cells number and height and increase interstitial tissue between tubules and in tramadol treated group, loss of most spermatogenic cells, degenerative changes, disturbed archeticture of seminiferous tubeules and edema of intersitial tissue.

\section{Conclusion}

Sildenafil and tramadol caused impaired function of testis in male rats but tramadol caused more significant decrease in sperm count and abnormal forms and more disturbed architecture of testis in histopathology. Tramadol also caused significant decrease in serum testosterone whereas sildenafil group showed normal testosterone level.

\section{References}

1. Boolell $M$, Allen $M J$, Ballard $S A$, et al. Sildenafil: an orally active type 5 cyclic GMP-specific phosphodiesterase inhibitor for the treatment of penile erectile dysfunction. Int J Impot Res. 1996; 8(2):47-52.

2. Moncada S, Higgs $A$. The L-arginine-nitric oxide pathway. N Engl J Med. 1993; 329(27):2002-12

3. Fisch JD, Behr B, Conti M. Enhancement of motility and acrosome reaction in human spermatozoa: differential activation by type-specific phosphodiesterase inhibitors. Hum Reprod. 1998; 13(5): 124854.

4. Morales A, Gingell C, Collins M, Wicker PA, Osterloh IH. Clinical safety of oral sildenafil citrate in the treatment of erectile dysfunction. Int J Impot Res. 1998; 10(2):69-74.

5. Marmor MF, Kessler R. Sildenafil (Viagra) and ophthalmology. Surv Ophthalmol. 1999; 44 (2):153-62.

6. Conti CR, Pepine CJ. Sweeney M. Efficacy and safety of sildenafil citrate in the treatment of erectile dysfunction in patients with ischemic heart disease. Am J Cardiol. 1999; 83 (5A):29-34

7. Raffa RB, Friderichs E, Reimann W, Shank RP, Codd EE, Vaught JL. Opioid and nonopioid components independently contribute to the mechanism of action of tramadol, an 'atypical' opioid analgesic. J Pharmacol Exp Ther 1992;260(1): ,275-85.

8. Spiller HA, Gorman SE, Villalobos D, et al. Prospective multicenter evaluation of tramadol exposure. J Toxicol Clin Toxicol. 1997; 35 (4),361-4.

9. Daubin C, Quentin C, Goullé JP, et al. Refractory shock and asystole related to tramadol overdose. Clin Toxicol (Phila). 2007;45 (8):961-4.

10. Lintz W, Erlaçin S, Frankus E, Uragg H. Biotransformation of tramadol in man and animal (author's transl). Drug Res.1981;31 (11): 1932-43.

11. Jellinek $\mathrm{H}$, Haumer $\mathrm{H}$, Grubhofer $\mathrm{G}$, et al. Tramadol in postoperative pain therapy. 
Patient-controlled analgesia versus continuous infusion. Anaesthesist. 1990; 39(10):513-20.

12. khalid G. effect of long term administration of sildenafil citrate (VIAGRA) on some sperm characteristics of male. Bas J Vet Res. 2009;8 (2):91.

13. El-Ghawet HA. Effects of tramadol on the reproductive function of wistar albino rats. European Journal of Experimental Biology. 2015; 5(1):56-64

14. Paget GE, Barnes JM. Toxicity tests in evaluation of drug activities pharmacometries (Laurence, D. R. and Bacharach, A. L. (Eds) Academic Press, London and New York, 1964.

15. Hardley ME. Endocrinology,2000. Prentice-Hall, Inc. PP. 585.

16. Burger M, Sikka SC, Bivalacqua TJ, Lamb DJ, Hellstrom WJ. The effect of sildenafil on human sperm motion and function from normal and infertile men. Int J Impot Res. 2000; 12(4):229-34.

17. Zavos PM, Zarmakoupis-Zavos PN. The use of sildenafil citrate for treatment of male infertility in males with erectile dysfunction. Obstet Gynecol. 2000; 95:54950.

18. Aversa A, Mazzilli F, Rossi T, Delfino M, Isidori AM, Fabbri A. Effect of sildenafil (Viagra) administration on seminal parameters and post-ejaculatory refractory time in normal males. Hum. Reprod. 2000; 15(1):131-4.

19. Jarrar BM.Histological alterations in the testicular tissue induced by sildenafil overdoses. Drug Metab Lett. 5(2):99-103.

20. Bliesener N, Albrecht S, Schwager A, Weckbecker K, Lichtermann D, Klingmuller D. Plasma testosterone and sexual function in men receiving buprenorphine maintenance for opioid dependence. J Clin Endocrinol Metab 2005; 90(1):203-6.

21. Reuhl J, Bachl $M$, Schneider $M$, Lutz $F$, Bratzke H. Morphometric assessment of testicular changes in drug related fatalities. Forensic Sci Int.2001; 115(3):171-81.

22. Sorge RE, Stewart J. The effects of longterm chronic buprenorphine treatment on the locomotor and nucleus accumbens dopamine response to acute heroin andcocaine in rats. Pharmacol Biochem Behav. 2006 ;84(2):300-5.

23. Yamamoto $\mathrm{Y}$, Yamamoto $\mathrm{K}$, Hayase $\mathrm{T}$, Abiru H, Shiota K, Mori C.Methamphetamine induces apoptosis in seminiferous tubules in male mice testis. Toxicol Appl Pharmacol.2002; 178(3): 155-60.

24. Ahmed MA. Kurkar A. Effects of opioid (tramadol) treatment on testicular functions in adult male rats: Role of oxidative stress: Role of nitric oxide and oxidative stress. Clin Exp Pharmacol Physiol.2014; 41(4):317-23 
